\title{
A study on the history of urban morphology in China based on discourse analysis
}

\author{
Limeng Zhang, Andong Lu \\ School of Architecture and Urban Planning, Nanjing University. Nanjing University, Nanjing, \\ China \\ E-mail: 554361151@qq.com, andonglu@gmail.com
}

\begin{abstract}
Urban morphology is a method widely used in China in the field of urban design and urban conservation. Since its first introduction to the Chinese context about 30 years ago, the key ideas and concepts of urban morphology underwent a significant phenomenon of 'lost in translation'. Different origins of morphological thoughts, different versions of translation, as well as different disciplinary context, have all together led to a chaotic discourse. This paper reviews the key Chinese articles in the field of urban morphology since 1982 and draws out a group of persistent keywords, such as urban form, growth mechanism, evolution and axis that characterize the morphological approach to urban issues, to find unusual evolutionary process. By reviewing the transformation of the definition of these keywords, this paper aims to generate an evolutionary diagram of landmark ideas and concepts.
\end{abstract}

Keywords: Urban morphology, terminology, discourse analysis

\section{Introduction}

Since the late 1970s, China has changed with a rapid urbanization. The development of hundreds of cities has undergone tremendous revolution in the restructuring of the economy and society. In this process, domestic scholars began to pay attention to the phenomenon of the city itself, trying to break through the traditional observation point of view, in order to re-understand the city and establish a new theoretical urban system. At the same time, the theory of urban morphology has been carried out for over 100 years, and initially set up a relatively comprehensive, clear observation mode, and effective analytical methods. Between the study of local urban theory and the application of western theory, 'urban form' came into being. In 1982, Kang Qi, the professor of architecture in Southeast University then, published 'The Form of the City--a Preliminary Study Outline', first proposed 'urban form' concept, marking the domestic scholars on the formal theory of urban morphology. Over the past 30 years, the urban morphology has gradually become a widely used method in the field of urban design and urban conservation in China, under the constant theoretical research and practical application in a large number of cases.

However, urban morphology characterizes in different school thoughts, different academic origins, and the long history of the development. In addition, factors that domestic research is lagging behind over abroad, and references of the translated versions are selected different, all lead into deviation of its main concepts, study methods, and analysis results. And in the process of localization there has been a confusion of terminology definitions, called 'lost in translation'. When Kang Qi (2011) reviewed urban form in his own theoretical system, he argued that, 'One of my graduate students back from the UK found it that the study of urban morphology has been lasting for a long period in European countries, such 
as Italy, Germany, Britain, France and so on, but my research was relatively earlier and continued in China.' Kang felt surprised about it because he didn't noticed it before. It shows that domestic development of urban form is originally independent from the European urban morphology, though it gradually interacted by the thoughts of urban morphology then and establishes its framework. Therefore, it is reasonable that some keywords are created in china's context, some are imported from abroad, and some are mixed together.

\section{Methodology}

It can be seen from relative articles published from the year of 1982 to the present that the study on urban morphology has a lot of branches by multiple methods. This article aims to select some representative pieces among literature, trying to interpret the evolutionary process with local characteristics of the terminology, and summing up the features of the phenomenon.

Regardless of its categories, taking articles using 'urban morphology' as keyword from the full-text journal database of CNKI as the basic statistics, the search result comes from 35 journals, reaching a total of 8866 articles.

The Figure 1 clearly shows the trend of the number of articles, which can be divided into three main phases. From 1982 to 1994, the study of urban morphology is still in the initial stage of exploration with a small quantity. From 1994 to 2006, theoretical research experienced a rapid growth period, reaching a relatively high level tenfold over the first phase. After 2006, the number of articles per year is above 500, and the study stays at maturity. The research contents also have different emphasis. The preliminary research focuses on the theoretical analysis, discussing the implication and influenced factors of urban form. Later scholars start to try to interpret the western theory of urban morphology, meanwhile constantly reviews the researches and summarizes the disciplines in domestic theories. In the mature period, 'urban morphology' has been regarded as a kind of inherent method to solve problems in city practice and application, combining with other disciplines and technical analysis methods.
The articles come from 30 disciplines, 35 journals, and more than half of which are from 9 authoritative publications. Over 100 authors contribute to the study, and many of them are well-known scholars, such as academicians form Chinese Academy of Engineering, headmasters and professors in architecture school, architects and urban planners. Considering the publishing year, citation times, the author's authority, the level of the journals and the research categories, this paper selects 32 representative articles as a basic sample, then extracted the key terms related to urban form from each article. These terms are often used frequently with the meaning explained more than one time, which clearly describes phenomenon in the context.

The Figure 3 shows that some of the keywords describe the constituent elements and characteristics of a city, which come from direct observation of the physical elements, such as block, plot, square, plan unit. Those words always have clear and certain definition among most of the articles, but compared with the Western theory, the recognition of its standards is different. For example, the concept of 'square' in the ancient Chinese city is relatively weak and insignificant. Square is an adjunctive place always attached to houses or roads, with the combination of traffic, trade, religious function. Square in Western context is much more formal and solemn. It is the occasion to held public speeches and other political campaigns, gathered a large number of religious and monumental architecture. The definition of 'important places of activity' has been confirmed already. Therefore, although definitions in the context of Chinese and foreign languages are partly similar, there are differences in the final appearance and function due to the different cultural background.

Another part of the key words is to sum up the reasons behind the urban morphology, trying to conclude characteristics of a sort of phenomenon, such as urban structure, evolution, spatial landscape, expansion. Those terms are often abstract summaries of certain features, or static descriptions of dynamic changes in time and space. Therefore, the definition of such terms is much vaguer 
than the first category. The larger scope, incomprehensive and insufficient description, result in misunderstanding of the terms, which needs supplement of other parts of the articles. As for the evolution of the deviation of the word meaning, this paper selected "urban morphology", "growth mechanism", "central axis" to explain how the change happens.

\section{Analysis}

\section{1.urban morphology}

The study of urban morphology is developed along two lines. One is rooted in the Chinese local city, still in the traditional field of urban research with a strong national style, called traditional concept. The other is from the Western theory, by translation and interpretation of the urban morphology and other urban theory or methodology, such as theories of urban functional structure, and typology. It reinterprets the Chinese city by the mature theoretical system which can be called foreign concept. The former one appeared much earlier while the latter one has a far more profound affection. Two lines developed from independently to collectively, explaining the concept of urban morphology from different perspectives.

When Kang Qi (1982) first proposed 'urban form', the core idea was that the urban form was inner and variable which means the internal implication of the structure is embodied in the external spatial form, and changes dynamically along with time. At the same time, he argued that the external characteristics of the standard is a superposition of economic, social, natural, science and technology, national, human, psychological, traffic and other elements in a certain period of time. This understanding can be considered as a relatively complete explanation of the concept. Kai Gu (2001) argued that the generalized urban morphology, including the social form and the physical environment, was a discipline under the influence of force for various urban activities (including political, social, economic and planning processes) without emphasizing the intrinsic logic of the city.
Kang Qi (1997) added new viewpoint to the research on the basis of the original study, not only emphasizing the inherent form, but also increasing the material and spiritual connotation on it. Meanwhile he put forward to the concept of 'cultural factor', and considered that the cultural factors from the historical events, social change, revolutionary resort will eventually affect the city itself. But this view was removed from the article in 2011, only reserving the concept in 1982.

Except for study of Kang Qi, in some other articles, urban morphology was regarded as a one-sided concept that consists partly of the structure, form, type or urban tissue in a city, often confused with the concept of layout. Instead of giving the urban form a definition, Liangyong $\mathrm{Wu}$ (1984) initiated the method of defining the city form with a general idea from several aspects, such as cityscape, layout, structure and so on. The "city form" refers to a series of urban components, such as walls, regional districts, businesses, sites. 'Style' in Chinese writing has two parts, in which the first character indicates local and traditional, and the other refers to the condition of the subject. The meaning of the style is too broad, often used to describe nature or custom. The 'layout' focuses on the relative location of different physical elements. The emphasis is on the feature of a city's structure, not enough to represent the overall meaning of a city's urban form.

As for the interpretation of Western theory, the definition of urban morphology has a certain common comprehension that different schools have different understanding. Two kinds of theories interact a lot in terminology, gradually reaching a consensus that the definition should be divided into broad and narrow sense. Besides Kai Gu (2001), Cui Zheng (2002) also believed that the general meaning of urban morphology refers not only to the urban parts of the tangible performance and geometric condition, but also a complex economic, cultural phenomena and social process, which is the result of interacting human activities and natural factors in a specific geographical environment and certain social and economic development. All of these definitions are influenced deeply by the study of Kang Qi, promoting its theory and 
the construction of the theoretical framework. Figure 4 is drawn by the number of references cited in Kang Qi, Kai Gu and Liangyong Wu. Three of them are the famous scholars in domestic urban theory research, but only citation of Kang Qi and Kai Gu's articles gradually increase during the period. It illustrates that the development is doomed to choose a more mature, perfect theoretical framework, and eliminate one-sided, vague thoughts.

\section{2.growth mechanism}

Growth mechanism is an original term in domestic research. It dates back early in time has a break in relative study. It origins in the meaning of internal implication (Kang Qi, 1982). Liu Qinghao (1995) cited Qi Kang's definition of urban form at the beginning of the studying growth mechanism, emphasizing that the connotation of "logic" and the extension of "performance" constitute the overall view of urban morphology together. Zhang Yuxing (1995) proposed that the growth mechanism is an important part urban morphology. He thinks that it embodies the dynamic growth of the form of self-dissipation and self-regulation, reflecting space, time, and human, which is the three most significant elements.

The growth mechanism essentially explores the inherent logic behind the changes in urban morphology. However, the logic behind comprises a complex theoretical system as well, composed of a mixture of factors together, and presenting differently. In this point, as a secondary category of urban morphology, the meaning of growth mechanism is great wider and more difficult to clarify than 'neighborhood', 'plot' and other physical features.

"Growth" refers to accretion and expansion from the center growing spot. There are three general situations: urban extension, land replacement, and city growth ring. Its growth pattern is divided into morphological growth and regional growth. The former is characterized by the type conversion, while the latter includes the expansion and transformation, generating 10 kinds of growth patterns.

Growth mechanism can not only be divided by performance and patterns, but also reflect the different stages of the urban morphology evolution process. When Cui Zheng (2002) concluded "the growth" according to articles from 1990, she suggested that "there is confliction of the old form and the new function, and then the old form gradually collapses while the new growth spot from the original form are free out ...... the new form develops to replace the old one and related adaptively to the new function. The evolution of the urban form is a dynamic process between accomplishment and adjustment.' Therefore, 'evolution' can be regarded as the ultimate consequence of growth mechanism, which is the appearance for the growth mechanism under the combined action of many factors.

According to the tendency of four terms, as the rapid growth of articles, the study of growth mechanism is further less than other words. Considering the reasons behind it, due to the excessive dimension of growth mechanism, it has not yet formed a unified definition and classification standard in the Chinese context, and it is not good as 'evolution' on reflecting the changes with time. It is gradually replaced by its various factors. However, the connotation of this term does not disappear, on the contrary, after breaking down into multiple terms, more accurate and detailed explanations of the principle exist in another form of the study.

\section{3.central axis}

Central axis is one of the important standards in the traditional urban research methods and design planning in china. The description is always accompanied by directions. Sicheng Liang promoted in 'History of Chinese Architecture' that those axis among China's ancient large buildings in the plane controlling the whole area are known as "central axis", and pointed out that it is only stressed mostly in China than any other countries in the world and has a prominent achievement. Liangyong Wu (1993) suggests in the plan of western district of Beijing that, designers should take good advantage of traditional method that first determine the central axis. For example, the plan should spread the axis northwards to the Fuhai lake in Yuanmingyuan Garden, southwards to Fengtai block, centered in 
Yuyuantan Park, and with ancient city and industrial zone on the both sides, green belt in the middle. Bochong Chen (1992) studies Fuzhou as another example. The design that building up a concrete high-rise building in the central axis among three great mountains severely damaged the layout of historic city. In the traditional sense, central axis is a sequence of nature, landscape, and architectural features. There is not only symmetrical residential distribution on this line, but also natural form of peaks, lakes, as well as palaces, temples and other landmarks. All elements can be regarded as a series of points, and central axis is the logic arrangement of the set of points under certain rules. This design principle reflects that the order in the traditional urban design occupies an important position.

Later the term of 'axis' occurs then as western theoretical research translated into domestic study. The axis is no longer central, iconic, political, and emphasizing on obvious belt area. Yuxing Zhang (1995) analyzed the axis along the river in Nanjing, and finally concluded that this area will form a new urban landscape belt. Chunlan Du (1998) mentioned Paris city developed along the Seine River axis. And Zhuojun Zou (2003) analyzed city of Hangzhou, considered that banded development close to natural green space which can create a good environmental condition, but if the belt city axis is too long, a series of urban problems will follow. Once axis means a region, it is no longer a straight-line shape. As long as it can indicate that the area is different from the surrounding environment, axis can also refer to the bending area, such as the river bank.

After that, the characteristics of the axis are further diluted. Jie Zhang (2003) mentioned in the large-scale urban design that the $20 \mathrm{~km}$ long urban road axis lacks the perception of actual space and time. Jiantao Zhang (2004) described a road in the urban regional planning. The urban street network is irregular, when land along the road expands to both sides, strengthening the road axis. In the two cases, 'Axis' only retains the meaning of the line. What it corresponds is more figurative, and more refers to a physical element with linear features, such as roads.
The evolution of the 'central axis' experienced deflecting. It is born in traditional design techniques, grows out large-scale city, and formulate a clear reference in modern design. The connotation simplified while the application is more extensive.

\section{Conclusion}

Urban morphology, growth mechanism and central axis represent three cases of evolution: fusion, separation and deflection. In addition, there are many other situations, such as substitution, combination, borrowing and so on. Figure 6 presents the evolution of key words.

The occurrence of 'lost in translation' in the research field of urban morphology, partly leads to a temporary misunderstanding of comprehension, but this phenomenon is not entirely negative and disordered. Although the domestic study only develops for a short period, the introduction of foreign theories and methods has accelerated this process, which has improved the depth and breadth of research in recent years. A large number of literature studies have formed a simple selecting model that retains the effective, precise, and popular concept, and meanwhile eliminates ambiguous, demise, obscure parts. The terms of the different sources are continually integrated, while some of which have been solidified by evolution, and some are cross-colliding in a variety of understandings to achieve precise levels and possibly create even new terms.

The meaning of the term is not static, even if the 'solidified concept' is also a 'temporary definition'. As the research discusses new observation, every term with certain meaning can be changeable to refer to new concepts. At the same time, with the paces of city development, new models, influencing factors and analytical techniques are needed in the study. Conceptual improvements or new vocabularies are inevitably generated. Thus, any term is likely to undergo a process of 'changeable - certain - changeable'. In the terms of the view, the phenomenon of terms evolution will be existing for a long time. 


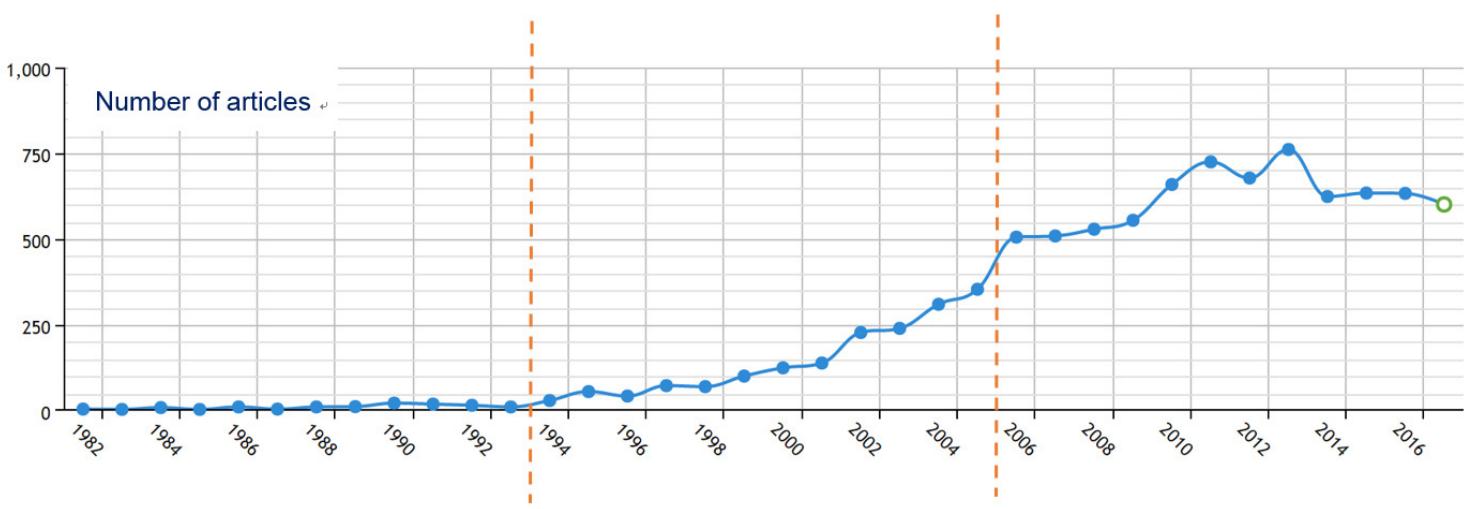

Figure 1. Articles using 'urban morphology' as keyword per year(www.cnki.net)

No.

$$
\text { Title }
$$

1 The Form of the City--a Preliminary Study Outline 2 The Layout Structure of Historic and Cultural City

3 The Propagation of Urban Physical Environment and Cultural Connotation

5 A Summary of City, Architecture and Space Structure Bochong Chen

Author

Kang Qi

Liangyong Wu

Bingren Xiang

Chunyu Wei

6 "Landscape City" and China's Urban Development in Liangyong Wu the 21st Century

7 Study on the Historical Characteristics and Evolvement Jianguo Wang Changshu City

8 Ecological Mechanism of Urban Form Qinghao Liu

9 The Elements and Processes of Urban Morphological Yuxing Zhang

Growth

Retrospect and Prospect of Research on Urban Fringe Jianming Zhang Belt

11 Context and Characteristics--Cultural Characteristics of Kang Qi

Urban Form

12 Study on Regional Characteristics and Urban Chunlan Du Morphology

Chunlan Du

Journal
City Planning Review
Architectural Journal
Architectural Journal
Huazhong Architecture
New Architecture
Architectural Journal
Journal of Southeast
University
City Planning Review
New Architecture
Human Geography
Urban Development
Studies
Journal of
Civil,Architectural \&
Environmental
Engineering

Publication

Time

$1982 / 12 / 27$

$1984 / 1 / 31$

Citation

$1988 / 11 / 26$

$1990 / 7 / 2$

1992/12/30

1993/6/30

1994/11/20

1995/3/9

1995/11/22

1997/9/30

1997/1/23

$1998 / 6 / 30$

Engineering

13 Study on the Formation and Form of the Fringe-belt $\quad x_{i}$ Wu, Hongjie Wu Huazhong Architecture

$2000 / 12 / 20$

City Planning Review

$2001 / 12 / 9$

City Planning Review

Cui Zheng, Lin Lin Exploring the Framework of Comprehensive and Rational Research

15 Review of Research on Urban Morphology in China since 1990

6 Urban Morphology Research and Spatial Strategic

Planning

Jin Duan City Planning Review

Discussion on the Evolution of Urban Morphology and Zhuojun Zou, Jianjun Planners the Dynamic Relationship Between Urban Water Yang

Systems
18 Study on the Chinese Traditional Urban Space Form Yigan from the View of the Theoretical Standard of Urban Yuan Form

19 From Large-scale City Design to the Daily Life Space

gang Zhou, Yue New Architecture

uan

Jie Zhang, Jie LV

City Planning Review

Jiantao Zhang

20 The Application of Urban Morphology
Planning of Historical Scenery Reserve

21 Theoretical Construction and Methods of Urban Landuse Patterns

Urban Development

Studies

22 The Basic Summary of the Evolution of Chinese Urban Morphology since the 1990

23 Application of Urban Morphology in the Renewal of Urban Marginal Area

24 A new Research Framework -- the Application of Urban Morphological Typology in China

Duan, Yulong

Guoping Qiong,

Dongfeng Yang.

Cong Guo

Tongtong Liu, Yuan

Zhan, Delong Sun

Fei Chen

South Architecture

Architectural Journal

Kang Qi

Modern Urban Research

$2002 / 7 / 9 \quad 352$

2003/2/9 $\quad 159$

$2003 / 2 / 25 \quad 63$

$2003 / 12 / 10 \quad 27$

2003/9/9 176

2004/11/20 52

2006/3/26 $\quad 8$

$2010 / 4 / 25 \quad 15$

$2010 / 2 / 28 \quad 0$

$2010 / 4 / 20 \quad 42$

$2011 / 5 / 15 \quad 20$

$2012 / 7 / 20 \quad 61$

2013/3/26 7

Hu, Pingping Dou

Sheng Yao, Jintang Urban Development Chen, Yinsheng Tian Studies

7 The Dilemma and Solution of Conzenian Urban

Morphological Regionalization Theory in China

Ying Zhou. Yinsheng Proceedings of 2013

Tian, Kai Wei China Urban Planning

Conference

Morphology Theory in Chin

Huifang Wang, Kai Progress in Geography

29 A Review of Chinese Urban Morphology in 2003-2013 Zhou

30 Experiences From Researches About Residential Areas Ji

Yhang Chen, Sheng Urban Development Employing Conzenian Approach

Yao, Yinsheng Tiang Urban De

31 Distinguishing Characteristics, Exploring Principles of Xu Li, Yue Che, City Planning Review Xenmei Zeng Hanmei Zeng Regional Uring

32 Use The Urban Morphology Theory Of Krier To Explain Gengnan Wei, Kang Architecture \& Culture The New Cities And Towns Development In China Qi

Figure 2.

Information

of articles

selected as

sample 


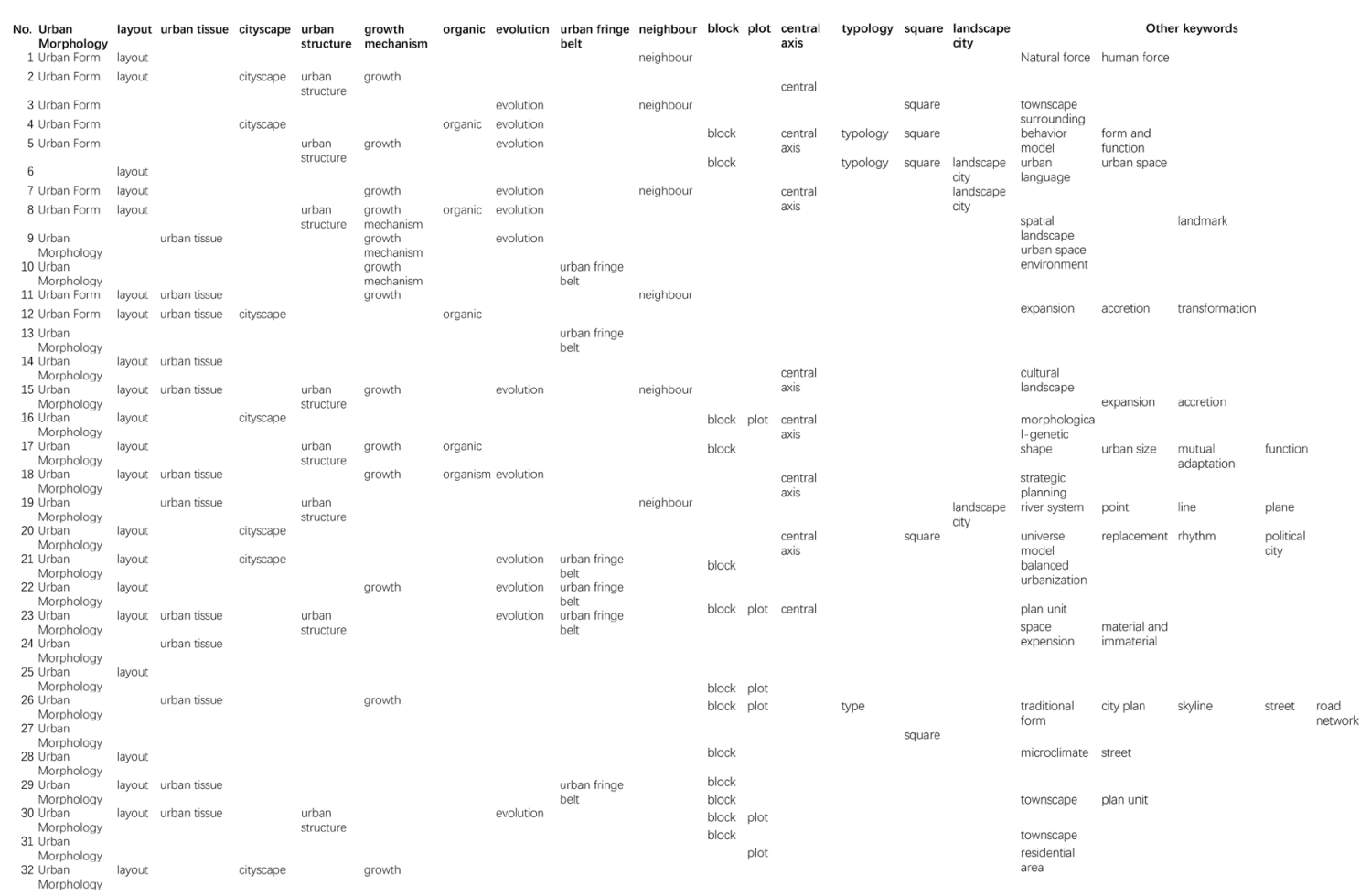

Figure 3. Key terms in sample articles(Number is the same as Figure 2

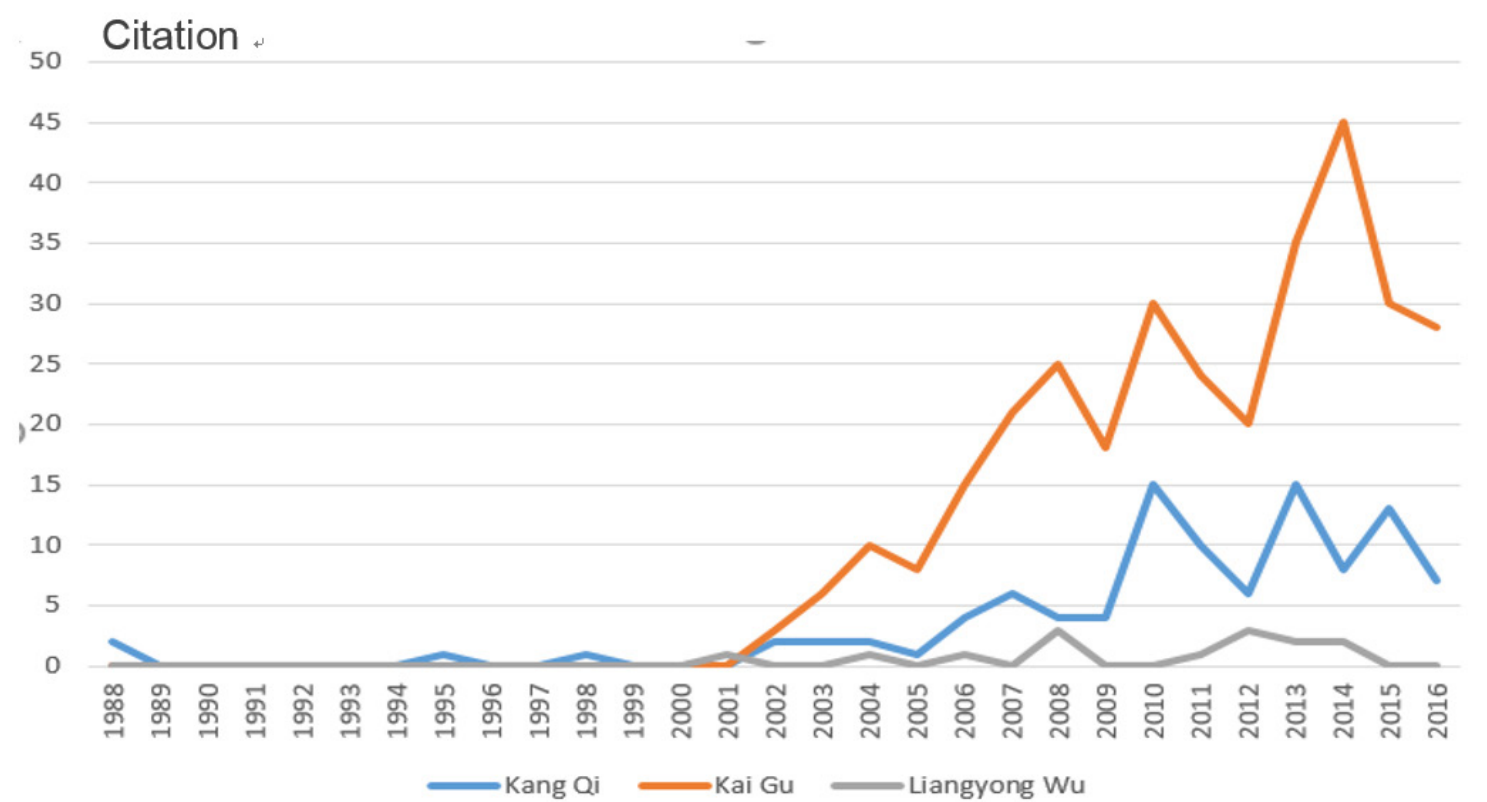

Figure 4. Citation of three well-known scholars`articles 

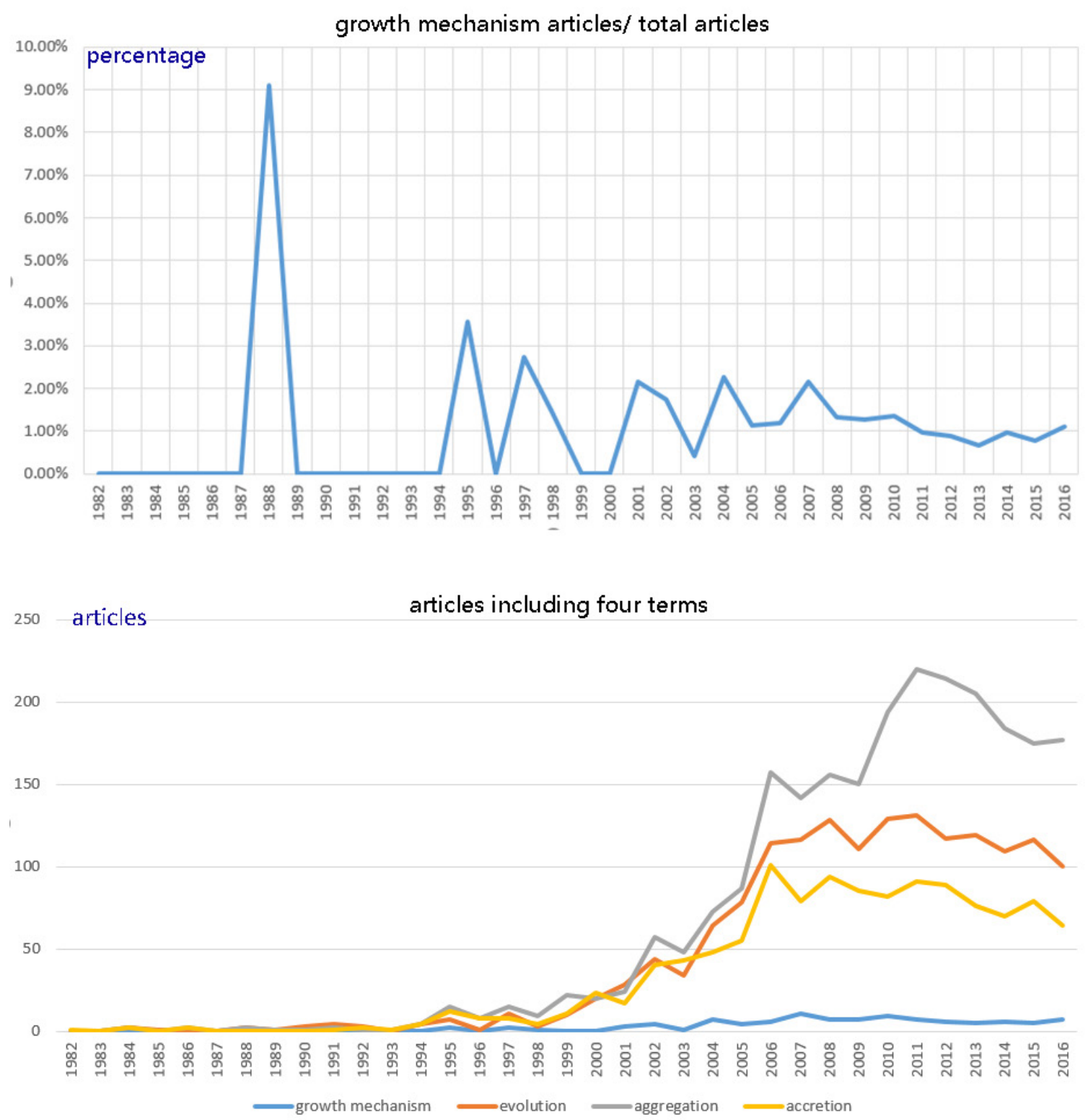

Figure 5. Development tendency of 'growth mechanism' 

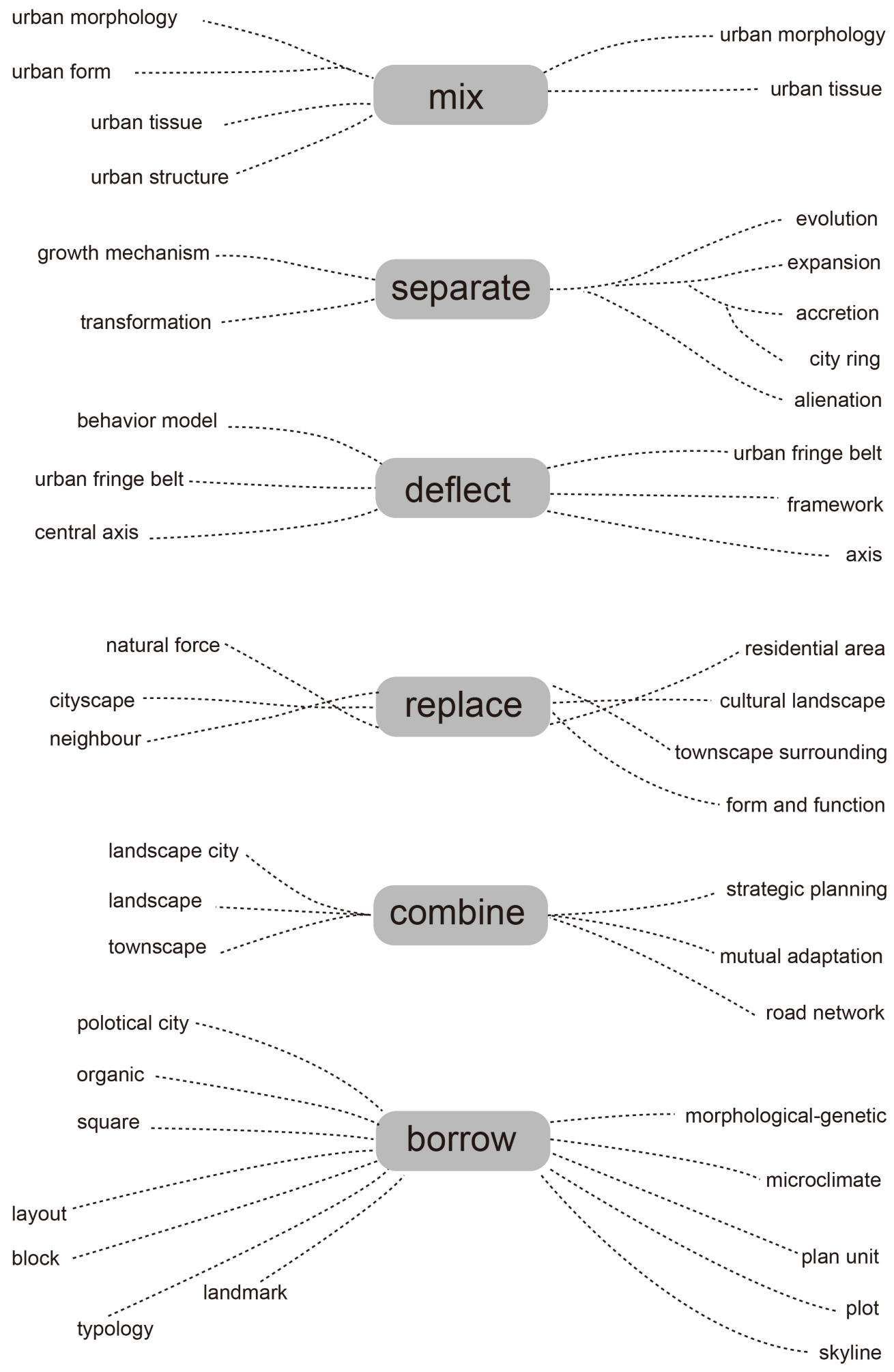

Figure 6. Diagram of key terms evolution 


\section{References}

Bochong Chen. (1992) 'A Summary of City, Architecture and Space Structure', New Architecture 04, 43-45.

Bingren Xiang. (1988) 'The Propagation of Urban Physical Environment and Cultural Connotation', Architectural Journal 11, 2427.

Chunlan Du. (1998) 'Study on Regional Characteristics and Urban Morphology', Journal of Civil,Architectural \& Environmental Engineering 03, 1-4.

Cui Zheng and Lin Lin. (2002) 'Review of Research on Urban Morphology in China since 1990', City Planning Review 07, 5964.

Fei Chen. (2010) 'A new Research Framework -- the Application of Urban Morphological Typology in China', Architectural Journal 04, 85-90.

Guoping Qiong and Dongfeng Yang and Jianxun Yu. (2010) 'The Basic Summary of the Evolution of Chinese Urban Morphology since the 1990s', Huazhong Architecture 04, 120-123.

Huifang Wang, Kai Zhou. (2014) 'A Review of Chinese Urban Morphology in 2003-2013 Years', Progress in Geography 05, 689-701.

Jintang Chen and Sheng Yao and Yinsheng Tian. (2014) 'Experiences From Researches About Residential Areas Employing Conzenian Approach', Urban Development Studies 02, 14-21.

Jin Duan. (2003) 'Urban Morphology Research and Spatial Strategic Planning', City Planning Review 02, 45-48.

Jianguo Wang. (1994) 'Study on the Historical Characteristics and Evolvement of Changshu City', Journal of Southeast University 06, $1-5$.

Kai Gu. (2001) 'The Theory and Method of Urban Morphology--Exploring the Framework of Comprehensive and Rational Research', City Planning Review 12, 36-42.

Kang Qi. (1982) 'The Form of the City--a Preliminary Study Outline', City Planning Review 06, 16-25.

Kang Qi. (1997) 'Context and Characteristics-Cultural Characteristics of Urban Form', Urban Development Studies 01, 1-5.
Kang Qi. (2011) 'The Form of the City', City Planning Review 05, 92-96.

Liangyong Wu. (1984) 'The Layout Structure of Historic and Cultural City', Architectural Journal 01, 22-26.

Qinghao Liu. (1995) 'Ecological Mechanism of Urban Form', City Planning Review 02, 20-22.

Sheng Yao and Jintang Chen and Yinsheng Tian. (2013) 'The Dilemma and Solution of Conzenian Urban Morphological Regionalization Theory in China', Urban Development Studies 03, 1-4.

Wowo Ding and Youpei $\mathrm{Hu}$ and Pingping Dou. (2012) 'Study on the Relationship between Urban Morphology and Urban Microclimate', Architectural Journal 07, 1621.

Yuxing Zhang. (1995) 'The Elements and Processes of Urban Morphological Growth', New Architecture 01, 27-30.

Zhuojun Zou and Jianjun Yang. (2003) 'Study on the Chinese Traditional Urban Space Form from the View of the Theoretical Standard of Urban Form', New Architecture 02, 87-90. 